\title{
Pflegebilder und Ethik - theoretische Grundlagen und Umsetzung in die Praxis
}

\author{
Sabine Ruppert
}

Dieser Beitrag bzw. der Vortrag zum Thema „Pflegebilder und Ethik“ basiert auf meinen Erfahrungen in verschiedenen Kontexten. Als diplomierte Gesundheits- und Krankenpflegerin und Pflegewissenschafterin bin ich in der Pflegepraxis, der Lehre, der Wissenschaft und der menschenrechtlichen Prävention tätig. In die Betrachtung der Pflegeethik und die Umsetzung in die tägliche Pflegepraxis fließen Beobachtungen, Erfahrungen und Berichte aus diesen Tätigkeitsfeldern sowie Ergebnisse aus internationalen Studien mit ein. Der Fokus dieses Beitrags liegt auf der Darstellung der wichtigsten Prinzipien, Werte und Normen der Pflegeethik und der Frage nach der Umsetzung dieser für die Pflegepersonen oft sehr theoretischen Konstrukte in die Praxis. Woran sollen sich Pflegepersonen orientieren? Wie können sie philosophisch-theoretische Überlegungen in den Alltag von Kliniken, Pflegeheimen und Hauskrankenpflege übertragen?

\section{Theoretische Grundlagen}

In diesem Abschnitt werden ausgewählte theoretische Grundlagen, die aus meiner Sicht bedeutend für die angewandte Ethik in der Pflege und damit auch für die Pflegepraxis sind, dargelegt. Einzelne ethische Aspekte werden durch persönliche Erfahrungen und Einschätzungen ergänzt.

\subsection{Menschenbild}

Es gibt verschiedene Arten von Menschenbildern, die auch Grundlage des Pflegeverständnisses sein können.

Im naturwissenschaftlichen Ansatz wird der Mensch als Maschine betrachtet, als funktionales Wesen. In der Medizin lässt sich beobachten, dass Ärztinnen und Ärzte immer mehr bzw. wieder dieses Menschenbild ihrer Tätigkeit zugrunde legen. Die heutige moderne Medizin beschäftigt sich im Klinikalltag vorwiegend mit Laborparametern, Zellen und Genen und es 
findet sich auch kaum mehr Zeit für umfassende Gespräche mit den betroffenen Menschen, um möglicherweise auch andere Gründe als rein physiologische bzw. pathologische Veränderungen für Erkrankungen zu erkennen.

In der Medizin wird auch das biopsychosoziale Menschenbild, bei dem Körper und Seele bzw. körperliche und psychische Vorgänge beachtet werden, propagiert. Im heutigen Gesundheitssystem müssen mittlerweile (Zeit-)Ressourcen geschaffen werden, um das ärztliche Handeln auch wirklich auf Basis dieses Menschenbildes umsetzen zu können.

Sowohl in der pflegerischen Ausbildung als auch in vielen Pflegeleitbildern wird das ganzheitliche Menschenbild, bei dem auf Ressourcen und Bedürfnisse der betroffenen Menschen eingegangen wird und das Zusammenspiel von Geist, Seele, Körper und sozialen Kontexten von Bedeutung ist, als Basis pflegerischen Handelns betrachtet. Es gelingt jedoch auch vielen Pflegepersonen nicht mehr, die betroffenen Menschen ganzheitlich zu betrachten. Spirituelle Aspekte werden häufig aus Angst vor Gesprächen über Tod und Sterben nicht angesprochen, soziale Aspekte werden als nicht wesentlich für die Betreuung im Kliniksetting betrachtet. Aufgrund von Personalbedarfsberechnungsmodellen besteht die Gefahr, dass der Fokus im Pflegeprozess auf die vier Bedürfnisbereiche (Körperpflege, Ausscheidung, Ernährung, Mobilisation) reduziert wird. Pflegepersonen sollten immer wieder ihr Handeln im Hinblick darauf, ob das ganzheitliche Menschenbild darin berücksichtigt wird, kritisch hinterfragen.

Weitere Menschenbilder, die auch für Pflegende Basis ihres Handelns sein können, sind beispielsweise:

- das humanistische Menschenbild mit der Orientierung an Interessen, Werten, Würde, Toleranz, Gewissens- und Gewaltfreiheit,

- das geisteswissenschaftliche Menschenbild mit der Erweiterung auf seelisch-geistige Lebensbezüge und

- das sozialwissenschaftliche Menschenbild, das davon ausgeht, dass wir nur in Gemeinschaft mit anderen Menschen leben und überleben.

Hinsichtlich der Abbildung des Menschenbildes im pflegerischen Handeln und der Reflexion des pflegerischen Handelns erscheint mir sinnvoll, dass sich Pflegepersonen sowohl am Beginn als auch immer wieder während ihrer jahrelangen Berufstätigkeit folgende Fragen stellen:

- Warum habe ich diesen Beruf gewählt?

- Wie will ich als Pflegeperson gesehen werden? 
- Was sind meine professionellen Werte?

- Wie sehe ich die Menschen, die ich pflege?

Erfolgt eine Reflexion anhand der oben genannten Fragen, so kann eine Auseinandersetzung mit dem eigenen Menschenbild und der eigenen Rolle als Pflegeperson stattfinden.

\subsection{Werte, Normen, Prinzipien}

Es werden nun einige ausgewählte Werte, Normen und Prinzipien näher erläutert. „Moralische Werte bilden einen Orientierungsrahmen für moralisches Handeln einer Person, innerhalb einer Gruppe oder Gesellschaft“ (Hiemetzberger 2016, 22). Werte sind bewusste oder unbewusste Orientierungsmaßstäbe und beschreiben Lebensinhalte, Handlungsziele oder Sinndeutungen. Eine festgelegte Form der Werterealisierung wird als Norm bezeichnet, d.h., Normen setzen die ihnen zugrunde liegenden Werte um. Prinzipien befinden sich wiederum eine Stufe darüber und stellen normative Orientierungspunkte dar - also Kriterien für richtiges und gutes Handeln. (Hiemetzberger 2016)

\subsubsection{Würde}

Die Würde des Menschen zu wahren, ist oberstes Prinzip allen pflegerischen Handelns. Würde kann als eine Wesenseigenschaft (absolute Würde) gesehen werden oder als Würde eines Menschen oder je bestimmter Menschen im Unterschied zu anderen (komparative Würde). (Marschütz, 2014) Aus theologischer Sicht gründet sich Würde in der Gottebenbildlichkeit und der Berufung zur Gemeinschaft mit Gott. Kant wiederum sieht Würde als inneren, absoluten Wert, begründet in Moralfähigkeit. Würde kann auch aufgrund sozialer Zuerkenntnis begründet oder als Gestaltungsauftrag gesehen werden. (Marschütz, 2014) Für die Wahrung der Würde im Pflegealltag ist es letztendlich nicht von großer Bedeutung, worin sich dies begründet. Pflegepersonen müssen bei verschiedenen Handlungen, wie z. B. der Unterstützung bei der Nahrungsaufnahme, der Körperpflege oder der Ausscheidung sowie bei der Kommunikation oder der Anwendung von medizinischen Maßnahmen, auf die Würde des betroffenen Menschen achten. 
Der Schweizer Berufsverband der Pflegenden (SBK) stellt fest, dass die Würde des Menschen und die Einzigartigkeit des Lebens im Zentrum allen pflegerischen Handelns stehen. (SBK 2008)

\subsubsection{Autonomie}

Ein weiteres wichtiges Prinzip, das es zu wahren gilt, stellt die Autonomie dar. Autonomie bedeutet selbstbestimmtes Entscheiden und bezieht sich auf die Fähigkeit des Menschen, für sich und sein Wohl entscheiden zu können. (Fölsch 2008)

Diese muss in allen pflegerischen Handlungen berücksichtigt werden. Auch zur Umsetzung der geplanten Pflegemaßnahmen sollen die Betroffenen vorab informiert und ihre Zustimmung eingeholt werden. Der Informed Consent, d. h. die informierte Zustimmung, gilt nicht nur für ärztliche bzw. medizinische Maßnahmen, sondern auch für pflegerische Handlungen. Dabei sind die Kompetenz (verstehen und entscheiden) und die Freiwilligkeit (der Entscheidung) von Seiten der Betroffenen eine Voraussetzung, auf Seiten der Pflegepersonen eine respektvolle Einstellung und respektvolles Handeln. Pflegepersonen müssen daher auch aufklären und Empfehlungen aussprechen bzw. eine selbstständige Entscheidung fördern. Die Betroffenen können selbst eine Entscheidung treffen oder eine andere Person dazu autorisieren. Selbstbestimmte Entscheidungen dürfen nicht durch lenkende Einflüsse oder Zwänge von außen verhindert werden. (Hiemetzberger 2013) Eine große Herausforderung stellt die Wahrung der Selbstbestimmung von kognitiv beeinträchtigten Menschen wie z. B. Menschen mit Demenz dar. Diese können möglicherweise nicht mehr autonom handeln, aber selbstbestimmt.

\subsubsection{Fürsorge}

Das ethische Prinzip der Fürsorge ist ein wichtiges Element der Pflege. Fürsorge orientiert sich an der Achtung der Würde des Menschen. Sie bedeutet zwischenmenschliche Beziehung und Verantwortung füreinander. Pflegepersonen bewegen sich oft in ethischen Konflikten zwischen der Wahrung der Autonomie der Betroffenen und der Umsetzung der pflegerischen Fürsorge. Dabei muss unbedingt beachtet werden, dass Fürsorge nicht zur Bevormundung werden darf. „Gut gemeint ist noch lange nicht gut", d.h. beispielsweise, was für mich als (Pflege-)Person gut ist bzw. 
scheint, muss nicht mit den Bedürfnissen des anderen Menschen übereinstimmen und darf niemandem aufgezwungen werden. Fürsorge wird oft gleichgesetzt mit dem Begriff Care, den Conradi folgendermaßen definiert:

"Care ist [...] eine Praxis der Zuwendung, Achtsamkeit und Bezogenheit, die durch die daran Beteiligten gemeinsam gestaltet wird. Gemeint ist ein weiter Bereich, der von Selbstsorge über kleine Gesten der Aufmerksamkeit, pflegende und versorgende menschliche Interaktionen bis hin zu kollektiven Aktivitäten reicht.“ (Conradi 2003, 32)

\subsubsection{Verantwortung}

In der Beschreibung von Fürsorge bzw. Care wird auf die Verantwortung füreinander verwiesen. Verantwortung im Kontext des Pflegealltags bedeutet die Zuständigkeit eines Subjekts der Verantwortung (Pflegeperson) für ein Objekt der Verantwortung (Betroffene) vor einem System von Bewertungsmaßstäben (Gesetz, Gewissen, Wissenschaft). Es wird zwischen moralischer Verantwortung, Rechtsverantwortung (GuKG) und der oben genannten Verantwortung für den anderen (Fürsorge) unterschieden. In der Praxis bedeutet dies für Pflegepersonen, Entscheidungen aufgrund ihrer fachlichen und ethischen Kompetenz zu treffen und entsprechend zu handeln. Für die Handlung und deren Auswirkungen müssen sie dann die Verantwortung übernehmen.

\subsection{Ethiktheorien}

Es gibt verschiedene Ethiktheorien, die auch in der Pflege zum Tragen kommen können.

Die Goldene Regel ist keine Theorie an sich, sondern stellt einen weit verbreiteten Grundsatz der praktischen Ethik dar, der in religiösen und philosophischen Texten etwa des Judentums, des Christentums und des Hinduismus aufscheint. Positiv formuliert wird die Goldene Regel beispielsweise im Neuen Testament: „Behandle andere so, wie du selbst behandelt werden willst.“ (Matthäus 7,12; Lukas 6,31) (Hiemetzberger 2013)

Aristoteles begründete die Tugendethik oder auch Strebensethik. Sie ist im vergangenen Jahrzehnt wieder ein zentrales Thema der Ethik geworden und beschreibt Grundhaltungen bzw. Charaktereigenschaften, durch die 
gutes Handeln möglich wird, wie z. B. die Kardinaltugenden Klugheit, Gerechtigkeit, Besonnenheit und Tapferkeit.

Immanuel Kant ist Begründer der Pflichtethik oder Normenethik bzw. Sollensethik. Dabei wird die Handlung an sich beurteilt. Oberstes Prinzip ist der Kategorische Imperativ: „Handle nur nach derjenigen Maxime, durch die du zugleich wollen kannst, dass sie ein allgemeines Gesetz werde!" (Kant 1974, 52)

Beim Utilitarismus, dessen Vertreter John Stuart Mill und Jeremy Bentham sind, steht das größtmögliche Glück für die größtmögliche Zahl an Menschen im Zentrum. Dies bedeutet, dass die Folgen einer Handlung beurteilt werden und nicht die Handlung an sich. Zentral ist das Prinzip der Nützlichkeit für alle Betroffenen, d. h. das allgemeine Wohlergehen (Sozialprinzip). (Hiemetzberger 2010, 2013; Arndt 2007)

Max Weber schuf den Begriff der Verantwortungsethik, zu deren Vertreter u.a. Hans Jonas zählt. Dabei geht es darum, Rechenschaft darüber abzugeben, warum eine bestimmte Entscheidung getroffen worden ist. Arndt spricht auch vom Prinzip der Verantwortung, das allen Menschen aufgetragen ist im Hinblick auf die Gefährdung unserer Welt und unsere eigene menschliche Verletzbarkeit. (Arndt 2007)

Eine besondere Stellung unter den Ethiktheorien nimmt die Care-Ethik bzw. Fürsorgeethik in der Pflege ein. Diese wird oft als synonym mit Pflegeethik betrachtet, jedoch kann Care-Ethik in verschiedenen Bereichen wie z. B. der Wirtschaft angewendet werden. Benner und Wrubel (1997) sehen Care als sorgende Haltung und Basis der Pflegepraxis (1989) an. Conradi (2001) bezeichnet Care als Ethik der Achtsamkeit.

\section{Pflegeethik}

Ethik in der Pflege gehört zur angewandten Ethik und ist die Reflexion moralischer Aspekte in den Handlungsfeldern der Disziplin Pflege: Pflegepraxis, Pflegemanagement, Pflegepädagogik, Pflegewissenschaft. (Lay 2004)

Pflegeethik ist wie die Medizinethik einem gesellschaftlichen Wertewandel unterlegen. So hat sich beispielsweise die Ansicht über die Anwendung freiheitsbeschränkender Maßnahmen wie das Anbringen von Seitenteilen innerhalb einer Generation von Pflegenden grundlegend geändert. War es früher ein „Pflegefehler“, die Seitenteile nicht hochzuziehen, wenn Pflegepersonen das Zimmer eines pflegebedürftigen, bettlägerigen Menschen verlassen haben, so ist diese Handlung nun nur mehr in äußerst seltenen Fällen fachlich angebracht und es gibt mittlerweile ausreichend alternative Maßnahmen. 
Joan Tronto (1993) beschreibt die Dimensionen der Pflege, die analog in den Schritten des Pflegeprozesses wiederzufinden sind:

- caring about: erkennen, dass jemand Pflegebedarf bzw. ein pflegerisches Problem hat,

- taking care of: sich des Problems annehmen,

- care giving: Fürsorge/Unterstützung/Pflege geben und

- care receiving: schauen, wie es bei Betroffenen ankommt.

Pflegethikerinnen und -ethiker orientieren sich an den vier Medizinethischen Prinzipien von Tom Beauchamp und James Childress (2009):

- Respekt vor der Autonomie von Personen (respect for autonomy),

- Prinzip der Wohltätigkeit (beneficience),

- Prinzip des Nicht-Schadens (nonmaleficence) und

- Gerechtigkeit (justice).

Sie fügen diesen Prinzipien andere hinzu, die sie für wichtig in der Pflegepraxis erachten oder formulieren Prinzipien um. Marianne Arndt (2007) nennt fünf Prinzipien einer Ethik der Verantwortung, nämlich:

- das Prinzip vom Wert des Lebens,

- das Prinzip des Guten und Richtigen,

- das Prinzip der Gerechtigkeit und Fairness,

- das Prinzip der Wahrheit und Ehrlichkeit und

- das Prinzip der individuellen Freiheit und Selbstbestimmung.

Marianne Rabe (2009) fügt den Prinzipien Autonomie, Gerechtigkeit und Fürsorge (Prinzip der Wohltätigkeit und des Nicht-Schadens) die Prinzipien des Dialogs, der Verantwortung und als übergeordnetes Prinzip die Würde hinzu. Reinhard Lay (2004) beschreibt fünf Prinzipien, nämlich:

- die Förderung von Wohlergehen/Wohlbefinden,

- die Förderung von Autonomie/Selbstständigkeit,

- die Gerechtigkeit,

- die Aufrichtigkeit und

- die dialogische Verständigung. 
Letzteres löst den Widerspruch zwischen dem Prinzip der Autonomie und dem Prinzip der Fürsorge.

Diese Prinzipienorientierung der Pflegeethik stößt jedoch auch auf Kritik. Chris Gastmans, Theologe aus Leuven, ist der Ansicht, dass die Prinzipien von Beauchamp und Childress sich auf eine Handlung, nämlich die Entscheidung zwischen „Leben und Tod“, beziehen sowie vor allem auf den klinischen Kontext und die Berufsgruppe der Ärztinnen und Ärzte. Pflege stellt jedoch einen Prozess dar und es geht um die ethischen Herausforderungen in der „alltäglichen Pflege“. Außerdem betreffen diese Handlungen nicht nur eine Berufsgruppe, sondern beziehen sich auf das ganze Gesundheitsteam - im institutionellen und gesellschaftlichen Kontext. Er konzipierte daher eine Health Care Ethics auf Basis des Personalismus und der Care-Ethik. Darin ist der Ausgangspunkt die Vulnerabilität der Betroffenen (erlebte Realität). Ziel der Pflege ist die Wahrung der Würde des Betroffenen (normativer Standard) mittels Care (interpretativer Dialog). Dieses Konstrukt gilt jedoch nicht nur für die Betroffenen, sondern auch für die Pflegepersonen. (Gastmans 2013)

\section{Transfer in die Praxis}

Nach all diesen theoretischen Erläuterungen stellt sich für die Pflegeperson in der Praxis die Frage, wie sie diese ethischen Normen, Werte und Prinzipien konkret in die Praxis umsetzen kann. Etwas konkretere Handlungsanweisungen geben die Menschenrechte und der ICN-Ethikkodex. Im ICN-Ethikkodex wird bereits in der Präambel ein Bezug zu den Menschenrechten hergestellt:

„Bei ihrer beruflichen Tätigkeit fördert die Pflegende ein Umfeld, in dem die Menschenrechte, die Wertvorstellungen, die Sitten und Gewohnheiten sowie der Glaube des Einzelnen, der Familie und der sozialen Gemeinschaft respektiert werden." (ICN-Ethikkodex für Pflegende 2014, 2)

Beide müssen letztendlich jedoch nochmals in die konkrete Praxis im Pflegealltag übersetzt werden. 


\subsection{Menschenrechte}

Die Menschenrechte werden in der Allgemeinen Erklärung der Menschenrechte der Generalversammlung der Vereinten Nationen festgehalten (Resolution 217A (III) der Generalversammlung 1948). Ebenso gibt es eine europäische Menschenrechtskonvention (Europäischer Gerichtshof für Menschenrechte 2003). Im Folgenden werden ausgewählte Artikel der Menschenrechtskonvention angeführt und in den pflegerischen Alltag transferiert.

Artikel 1 der Allgemeinen Erklärung der Menschenrechte stellt fest, dass alle Menschen frei und gleich an Würde und Rechten geboren sind. Dies gilt für alle Menschen, da jeder Anspruch hat

auf die in dieser Erklärung verkündeten Rechte und Freibeiten ohne irgendeinen Unterschied, etwa nach Rasse, Hautfarbe, Geschlecht, Sprache, Religion, politischer oder sonstiger Überzeugung, nationaler oder sozialer Herkunft, Vermögen, Geburt oder sonstigem Stand. (Artikel 2)

Die Wahrung der Würde ist - wie schon oben erwähnt - zentral bei allen Pflegetätigkeiten. Bereits in der Kommunikation mit den und über die betroffenen Menschen kann es zu Würdeverletzungen kommen. Nicht nur die direkte Kommunikation mit den Betroffenen sollte wertschätzend sein, sondern auch die Kommunikation über sie, z. B. bei der Dienstübergabe, der Pflegevisite oder in der Dokumentation. (Ruppert 2013)

Weitere Menschenrechte, die auch für die Pflegepraxis relevant sind:

Jeder hat das Recht auf Leben, Freiheit und Sicherheit der Person. (Artikel 3)

Dieser Artikel kann durch die unsachgemäße und überschießende sowie nicht nötige Anwendung von freiheitsbeschränkenden Maßnahmen, wie körpernahe Fixierung, Verwendung von Seitenteilen oder räumliche Einschränkung, verletzt werden. (Ruppert 2013)

Niemand darf der Folter oder grausamer, unmenschlicher oder erniedrigender Behandlung oder Strafe unterworfen werden. (Artikel 4)

In Bezug auf Artikel 4 gibt es leider auch Berichte von Pflegepersonen, die den Menschen, die sie betreuen und pflegen, Schaden zufügen, z. B. durch absichtliches langes Stehenlassen beim Bettenmachen oder durch Duschen mit kaltem Wasser. (Ruppert 2013)

Alle Menschen sind vor dem Gesetz gleich und haben obne Unterschied Anspruch auf gleichen Schutz durch das Gesetz. Alle haben Anspruch auf glei- 
chen Schutz gegen jede Diskriminierung, die gegen diese Erklärung verstößt, und gegen jede Aufhetzung zu einer derartigen Diskriminierung. (Artikel 7)

Dieser Artikel trifft allgemein auf die Menschen zu, die Pflege erhalten. Sie sollten alle unabhängig von persönlichen Merkmalen wie z.B. Geschlecht, Alter, Herkunft, sexueller Orientierung usw. gleichbehandelt werden.

Jeder hat das Recht auf Gedanken-, Gewissens- und Religionsfreibeit; dieses Recht schließt die Freiheit ein, seine Religion oder seine Weltanschauung zu wechseln, sowie die Freiheit, seine Religion oder seine Weltanschauung allein oder in Gemeinschaft mit anderen, öffentlich oder privat durch Lehre, Ausübung, Gottesdienst und Kulthandlungen zu bekennen. (Artikel 18)

Dieser Artikel beinhaltet u. a. das Ermöglichen der Religionsausübung und den Respekt vor den entsprechenden Bräuchen und Sitten, die auch Auswirkungen auf Ernährung, Umgang mit Angehörigen und Sterbebegleitung haben können. (Ruppert 2013)

Jeder hat das Recht auf Meinungsfreiheit und freie Meinungsäußerung. (Artikel 19)

Eine Grundvoraussetzung bei der Beachtung dieses Artikels ist die umfassende und verständliche Information der betroffenen Menschen. Sie sollen in die medizinischen und pflegerischen Entscheidungen miteinbezogen werden, z.B. in die Erstellung von Pflegediagnosen und die Umsetzung des Pflegeprozesses. (Ruppert 2013)

Jeder hat als Mitglied der Gesellschaft das Recht auf soziale Sicherheit und Anspruch darauf, durch innerstaatliche Maßnabmen und internationale Zusammenarbeit sowie unter Berücksichtigung der Organisation und der Mittel jedes Staates in den Genuss der wirtschaftlichen, sozialen und kulturellen Rechte zu gelangen, die für seine Würde und die freie Entwicklung seiner Persönlichkeit unentbebrlich sind. (Artikel 22)

Jeder hat das Recht auf einen Lebensstandard, der seine und seiner Familie Gesundheit und Wobl gewährleistet, einschließlich Nahrung, Kleidung, Wohnung, ärztliche Versorgung und notwendige soziale Leistungen, sowie das Recht auf Sicherheit im Falle von Arbeitslosigkeit, Krankheit, Invalidität oder Verwitwung, im Alter sowie bei anderweitigem Verlust seiner Unterhaltsmittel durch unverschuldete Umstände. Mütter und Kinder haben Anspruch auf besondere Fürsorge und Unterstützung. (Artikel 25)

Artikel 22 und 25 erhalten vor allem in der Langzeitbetreuung Relevanz. Ausreichend Kontakt zu Angehörigen muss ermöglicht werden. Soziale 
Aktivitäten werden auf die Wünsche und Ansprüche der Betroffenen abgestimmt und in einem entsprechenden Ausmaß angeboten. (Ruppert 2013)

\subsection{ICN-Ethikkodex}

Berufskodizes bilden ein zusammenhängendes Ganzes von ethischen Prinzipien und Regeln bezüglich der Ziele und Werte eines Berufes sowie der Haltung und des Verhaltens, die für das Fördern und Evaluieren des beruflichen Handelns notwendig sind. (Van der Arend/Gastmanns 1996) Ein Berufskodex gibt der Gesellschaft gegenüber Auskunft über Werte und Normen, an denen eine Berufsgruppe ihr Handeln ausrichtet. (Hiemetzberger 2010) Der ICN-Ethikkodex beschreibt vier Aufgaben der Pflegenden:

- Gesundheit fördern,

- Krankheit verhüten,

- Gesundheit wiederherstellen und

- Leiden lindern.

Weiters werden ethische Verhaltensweisen zu vier Elementen genannt, nämlich den Elementen:

- Pflegende und ihre Mitmenschen,

- Pflegende und die Berufsausübung,

- Pflegende und die Profession sowie

- Pflegende und ihre Kolleginnen bzw. Kollegen.

Diese stellen einen Rahmen für die ethische Qualität pflegerischen Handelns und Verhaltens dar.

Ein wichtiger Abschnitt des ICN-Ethikkodex ist schon die Präambel, in der ethische Grundsätze angeführt werden:

„Untrennbar von Pflege ist die Achtung der Menschenrechte, einschließlich kultureller Rechte, des Rechts auf Leben und Entscheidungsfreiheit auf Würde und auf respektvolle Behandlung. Pflege wird mit Respekt und ohne Wertung des Alters, der Hautfarbe, des Glaubens, der Kultur, einer Behinderung oder Krankheit, des Geschlechts, der sexuellen Orientierung, der Nationalität, der politischen Einstellung, der ethnischen Zugehörigkeit oder des sozialen Status ausgeübt. Die Pflegende übt ihre berufliche Tätigkeit zum Wohle des 
Einzelnen, der Familie und der sozialen Gemeinschaft aus; sie koordiniert ihre Dienstleistungen mit denen anderer beteiligter Gruppen." (ICN-Ethikkodex 2014, 1)

\section{Herausforderungen}

Auch wenn es den Anschein hat, dass es genug ethische Theorien, Werte, Normen und Prinzipien sowie Regelwerke wie die Menschenrechte und den ICN-Ethikkodex zur Orientierung im Pflegealltag gibt, kann es für die einzelne Pflegeperson noch immer schwierig sein, diese hohen ethischen Ansprüche in die Praxis umzusetzen.

Pflegepersonen sollen eine ethische Kompetenz aufweisen, die aus Zuwendung (caring about), Verantwortung, moralischer Sensitivität, Anwesenheit bzw. Achtsamkeit, Selbstreflexion und Dialog besteht. Das reflektierte Wissen, die praktische Weisheit und das begründete Wissen bilden die ethische Sensibilität. Jedoch muss festgestellt werden, dass beides keine Garantie darstellt, um moralische Handlungskompetenz entwickeln zu können

Moralische Handlungskompetenz bedeutet, dass Pflegepersonen eine eigenständige Einstellung zu „richtigem“ und „falschem“ pflegerischen Handeln besitzen und Verantwortung für ihr Handeln übernehmen.

Wenn jedoch die ethische Kompetenz sowie die ethische Sensibilität vorhanden sind, aber externe Faktoren wie z. B. das Fehlen von Ressourcen die Pflegepersonen daran hindern, ihr Bestes zu tun, kann moralischer Distress oder, wie Karin Kersting (2011) es nennt, Coolout entstehen.

In einem aktuellen Artikel zu moralischem Distress unter Intensivpflegepersonal werden folgende Gründe für dessen Auftreten genannt: Zusammenarbeit zwischen Pflegepersonen und Ärztinnen/Ärzten; Umgebungsfaktoren, ethisches Klima; Konfliktlösung; Beziehungen im Pflegeteam; Autonomie der Pflegepersonen; psychologisches Empowerment der Pflegepersonen; End-of-life-decisions-Prozess; Kommunikation; Unterstützung durch Vorgesetzte; Personalressourcen; hierarchische Beziehungen; schlechte Teamarbeit; inkompetente Angehörige von Gesundheitsberufen; Gewalt (beidseitig), fehlende Unterstützung; schlechte technische Ausstattung; Arbeitsbelastung usw.

Dies alles kann negative Folgen sowohl für die Pflegepersonen als auch für Betroffene und deren Bezugspersonen haben. Auf Seiten der Pflegepersonen können ein Arbeitswechsel, Berufsausstieg und Burn-out die Folgen sein. Auf Seiten der Betroffenen können mangelnde Kommunikation, verlängerte Behandlungs- und Sterbeprozesse, inadäquate pflegerische Unter- 
stützung, Depersonalisierung des Betroffenen, Kontaktabnahme zu Betroffenen und Bezugspersonen, schlechte Lebensqualität usw. auftreten. (McAndrew et al. 2018)

Um die moralische Handlungskompetenz der Pflegepersonen heranzubilden und sie bei der Umsetzung im Pflegealltag zu unterstützen, sind fachliche und ethische Bildung nötig. Genauso wichtig ist aber die Organisationsethik, die verhindert, dass es zu Moral Distress unter den Pflegepersonen kommt. Ein weiterer wichtiger Aspekt ist die gesellschaftliche Verantwortung, d.h. die Moral in einer Gesellschaft. Diese kann über die Gesetzgebung Einfluss auf die Pflegeethik haben. Ebenso kann die gesellschaftliche Moral den Umgang mit bestimmten Gruppen von Menschen wie z. B. alten Menschen bestimmen.

Hinsichtlich der ethischen Bildung stellt sich immer wieder die Frage, welche Art von Ethikunterweisung die sinnvollste ist. Im RIPE-Projekt wurde diese Frage erforscht und Gallagher et al. (2016) kamen zum Schluss, dass konventionelle Bildung (face to face), reflektorische ethische Bildung (reflective groups) und die Eigenerfahrung (z. B. immersive simulation im sTimulmodel2) zur Heranbildung von moralischer Handlungskompetenz sinnvoll erscheinen. (Gallagher et al. 2016; Gallagher 2016) Abschließend möchte ich auf ein Zitat von Liliane Juchli verweisen:

„Pflege beruht auf Achtung und Ehrfurcht gegenüber dem anderen Menschen. Sie orientiert sich an der Würde der Person. Diese Achtung wie auch der Respekt vor dem Anderen sind im konkreten Handeln ebenso zu erkennen wie in der Sprache, derer wir uns bedienen." (Von Fellenberg-Bitzi 2013, 130) 


\section{Literatur}

Arndt, Marianne: Ethik denken. Maßstäbe zum Handeln in der Pflege, Stuttgart: Thieme ${ }^{2} 2007$.

Beauchamp, Tom/Childress, James: Principles of Biomedical Ethic, Oxford: Oxford University Press ${ }^{6} 2009$.

Benner, Patricia/Wrubel, Judith: Pflege, Stress und Bewältigung. Gelebte Erfahrung von Gesundheit und Krankheit. Aus dem Amerikanischen übersetzt von Irmela Erckenbrecht. Bern: Hans Huber 1997.

Conradi, Elisabeth: Take Care. Grundlagen einer Ethik der Achtsamkeit, Frankfurt/Main: Campus 2001.

Conradi, Elisabeth: Vom Besonderen zum Allgemeinen - Zuwendung in der Pflege als Ausgangspunkt einer Ethik, in: Wiesemann, Claudia/Erichsen, Norbert/ Behrendt, Heidrun/Biller-Adorno, Nikola/Frewer, Andreas (Hg.): Pflege und Ethik. Leitfaden für Wissenschaft und Praxis, Stuttgart: Kohlhammer 2003, 3046.

Europäischer Gerichtshof für Menschenrechte: Konvention zum Schutze der Menschenrechte und Grundfreiheiten (2003), in: http://www.bmask.gv.at/cms/site/at tachments/3/3/9/CH2255/CMS1219127809211/europaeische_menschenrechtsko nvention.pdf [27.9.2013].

Fölsch, Doris: Ethik in der Pflegepraxis, Wien: Facultas 2008.

Gallagher, Ann: What counts as 'ethics education'? in: Nursing Ethics 23/2 (2016) Editorial.

Gallagher, Ann/Peacock, Matthew/Zasada, Magdalena/Coucke, Trees/Janssens, Nele: Care-givers' reflections on an ethics education immersive simulation care experience. A series of epiphanous events, in: Nursing Inquiry 24/3 (2016) e12174.

Gastmans, Chris: Dignity-enchancing nursing care. A foundational ethical framework, in: Nursing Ethics 20/2 (2013) 142-149.

Hiemetzberger, Martina/Messner, Irene/Dorfmeister, Michaela: Berufsethik und Berufskunde, Wien: Facultas ${ }^{2} 2010$.

Hiemetzberger, Martina: Ethik in der Pflege, Wien: Facultas 2013.

ICN-Ethikkodex für Pflegende 2014, in: https:/www.pflege-charta.de/fileadmin/ch arta/Arbeitshilfe/Modul_5/M5-ICN-Ethikkodex-DBfK_.pdf [16.2.2017].

Kant, Immanuel: Kritik der praktischen Vernunft. Grundlegung zur Metaphysik der Sitten. Werkausgabe Band VII, Hg. von Wilhelm Weischedel, Frankfurt/Main: Suhrkamp 1974.

Kersting, Karin: „Coolout“ in der Pflege. Eine Studie zur moralischen Desensibilisierung, Frankfurt: Mabuse 2011.

Lay, Reinhard: Ethik in der Pflege. Ein Lehrbuch für die Aus-, Fort- und Weiterbildung, Hannover: Schlütersche Verlagsgesellschaft 2004.

Marschütz, Gerhard: Theologisch ethisch nachdenken. Band 1: Grundlagen, Würzburg: Echter 2014. 
McAndrew, Natalie Susan/Leske, Jane/Schroeter, Kathryn: Moral distress in critical care nursing. The state of the science, in: Nursing Ethics 23/5 (2018) 552-570.

Rabe, Marianne: Ethik in der Pflegeausbildung. Beiträge zur Theorie und Didaktik, Bern: Huber 2009.

Resolution 217 A (III) der Generalversammlung: Allgemeine Erklärung der Menschenrechte (1948) in: http://www.ohchr.org/EN/UDHR/Pages/Language.aspx?L angID=ger [27.9.2013].

Ruppert, Sabine: Menschenrechte in der Pflege. Die Achtung von Würde und Autonomie der PatientInnen, in: Pflegenetz 5 (2013) 12-14.

SBK (Schweizer Berufsverband der Pflegefachfrauen und Pflegefachmänner): Ethik in der Pflegepraxis, Bern: SBK 1990, 2003, Nachdruck 2008.

Tronto, Joan: Moral Boundaries. A political Argument for an Ethic of Care, New York: Routledge 1993.

Valizadeh, Leila/Zamanzadeh, Vahid/ Dewar, Belinda/Rahmani, Azad/Ghafourifard, Mansour: Nurse's perceptions of organisational barriers to delivering compassionate care. A qualitative study, in: Nursing Ethics 23/5 (2018) 580-590.

Van der Arend, Arie/Gastmans, Chris: Ethik für Pflegende, Bern: Huber 1996.

Von Fellenberg-Bitzi, Trudi: Liliane Juchli - ein Leben für die Pflege, Stuttgart/New York: Georg Thieme Verlag 2013. 
